\title{
The Spin-1/2 Ising Model with Skew Magnetic Field at High Temperatures
}

\author{
E.V. Corrêa Silva ${ }^{1}$, S.M. de Souza ${ }^{2}$, Onofre Rojas ${ }^{2}$, James E.F. Skea ${ }^{3}$, and M.T. Thomaz ${ }^{4}$ \\ ${ }^{1}$ Departamento de Matemática e Computação, Faculdade de Tecnologia, \\ Universidade do Estado do Rio de Janeiro. Estrada Resende-Riachuelo, s/ $n^{o}$, \\ Morada da Colina, CEP 27523-000, Resende-RJ, Brazil \\ ${ }^{2}$ Departamento de Ciências Exatas, \\ Universidade Federal de Lavras, \\ Caixa Postal 3037, CEP 37200-000, Lavras-MG, Brazil \\ ${ }^{3}$ Departamento de Física Teórica, Instituto de Física, \\ Universidade do Estado do Rio de Janeiro, \\ R. São Francisco Xavier $n^{0} 524$, \\ CEP- 20559-900, Rio de Janeiro-RJ, Brazil \\ ${ }^{4}$ Instituto de Física, Universidade Federal Fluminense, \\ Av. Gal. Milton Tavares de Souza $s / n^{o}$, \\ CEP 24210-346, Niterói-RJ, Brazil
}

Received on 26 October, 2007

\begin{abstract}
We calculate the thermodynamics of the one-dimensional spin-1/2 Ising model in the presence of a constant skew magnetic field. We obtain the high-temperature expansion of its Helmholtz free energy (HFE), for the ferromagnetic and antiferromagnetic cases, up to order $\beta^{7}$. This expansion permits us to obtain the behaviour of the model for $|J| \beta \lesssim 1$, when it cannot be described by its classical version. Among the calculated thermodynamical functions of the model, we have the diagonal elements of the magnetic susceptibility tensor for the transverse and logitudinal Ising models, obtained by taking the limits $h_{z} \rightarrow 0$ and $h_{y} \rightarrow 0$, respectively, of the $\beta$-expansion of the HFE. The $y$-component of the magnetization and the $\chi_{y y}$ component of the magnetic susceptibility tensor are almost the same for the antiferro- and ferromagnetic models, at least for $|J| \beta \lesssim 1$; and, $\chi_{y y}$ is practically independent of the direction of the external magnetic. We also show that, in this region of temperature, the thermodynamics of the Ising model with skew magnetic field and that of an $X X Z$ model with longitudinal magnetic field are not similar.
\end{abstract}

Keywords: Quantized spin models; Spin chain models; Lattice theory and Statistics of Ising model; High temperature expansion

\section{INTRODUCTION}

The spin-1/2 Ising model with nearest-neighbor interactions is certainly one of the simplest one-dimensional class of models. In spite of its simplicity, it has some agreeable features. For either a purely longitudinal or purely transversal external magnetic field, it becomes exactly solvable. The exact thermodynamics of the longitudinal case was calculated in 1925 by Ising in his original paper[1]. In 1941 Kramers and Wannier[2] reobtained the exact thermodynamics of the Ising model in the presence of a longitudinal magnetic field by using the transfer matrix approach extended to the planar model. Pfeuty[3] derived in 1970 the exact thermodynamics of the transversal Ising model, calculating its free energy; he showed the equivalence of this model to a system of noninteracting fermions, by a set of suitable transformations. One interesting aspect of the transversal model is its conformal invariance for a finite interval of temperature (including $T=0 \mathrm{~K}$ ), in which it has a quantum critical point (QCP)[4]. We should mention that the exact HFEs of the Ising model with longitudinal $\left(h_{y}=0\right)$ or transversal $\left(h_{z}=0\right)$ constant magnetic fields are not enough to determine all the elements of the magnetic susceptibility tensor; a more complete picture requires information on intermediary orientations of the magnetic field with respect to the easy-axis.
However the one-dimensional Ising model in the presence of a magnetic field with both longitudinal and transversal components (a skew magnetic field) is not exactly soluble: the presence of both components of the magnetic field simply destroys its integrability. As an unhappy consequence, the powerful "Bethe ansatz"[5] cannot yield the exact thermodynamics of the model. The ferromagnetic version of the model, at $T=0 \mathrm{~K}$, was studied by Fogedby[6] in the seventies. More recently, Ovchinnikov et al. studied the phase diagram, at zero temperature, of the Ising model in the presence of a skew magnetic field[7] and showed the existence of a critical line that separates the antiferromagnetic and paramagnetic states. The classical limit of this model also presents a critical line between these two states.

Among the new materials intensively researched in the last decade, the so-called single-chain magnets (SCM) exhibit strong uniaxial anisotropy at nanometric scale, thus representing the possibility of high-temperature metastable magnetic properties that would qualify those materials as prototypes for molecular memory devices. In particular, the heterometallic $\mathrm{Mn}^{I I I}$ and $\mathrm{Ni}^{I I}$ chain is an example of a SCM with onedimensional behaviour up to very low temperatures[8]; there is a region of temperature for which its local intra-trimer excitations are inactive, and the effective ferromagnetic chain behaviour that arises can be described by the one-dimensional 
spin-1/2 Ising model. It is certainly interesting to have analytic expansions of the thermodynamical functions of the Ising model to fit the data for arbitrary orientation of the external magnetic field. This kind of knowledge permits looking for new features of the material at finite temperatures.

The aim of the present communication is to study the thermodynamics of both the ferromagnetic and antiferromagnetic cases of the one-dimensional spin-1/2 Ising model in the presence of a skew magnetic field in the region of temperature of $|J| \beta \lesssim 1$, where $\beta=1 / k T, k$ is the Boltzmann constant, $T$ is the absolute temperature in Kelvin and $J$ is the coupling strength between first-neighbour $z$-components of spin (see the Hamiltonian (1)).

In section II we present the Hamiltonian of the onedimensional spin-1/2 Ising model in the presence of a mixed magnetic field. In section III we study the thermodynamics of this model by applying the method of Ref. [9] directly to the Hamiltonian (1). The thermodynamical functions of the model depend on the sign of $J /|J|$, on the norm of the magnetic field, on its angle $\theta$ with respect to the easy-axis, and on the temperature. One of our aims is to explicitly verify if for $|J| \beta \lesssim 1$ there is any trace of the at $T=0 \mathrm{~K}$ phase transition of the antiferromagnetic model. In subsection III A such eventual trace is sought by fixing the norm of the magnetic field and varying the angle $\theta$. Its norm is such that for $T=0 \mathrm{~K}$ and $\theta \in\left[0, \theta_{C}\right]$, the system is in the antiferromagnetic state, and for $\theta$ larger than the critical angle $\theta_{C}$ (still at $T=0 \mathrm{~K}$ ) the system crosses the critical line of the phase diagram, presented in Ref. [7]. In this subsection we consider the behaviour of various thermodynamical quantities as functions of $\theta$ for a constant temperature. In subsection III B, we study the dependence of the thermodynamical functions on the temperature and the norm of the magnetic field and present a new duality between the transversal Ising model and the $X Y$ model. We also verify if the similarity of the Ising model with skew magnetic field and the $X X Z$ model with longitudinal magnetic field survives in the region of high temperatures. In section IV we present our conclusions. Appendix A contains the high temperature expansion of the HFE for the spin-1/2 Ising model in the presence of a mixed constant external magnetic field, up to order $\beta^{7}$.

\section{THE SPIN-1/2 ISING MODEL WITH SKEW MAGNETIC FIELD}

The Hamiltonian of the one-dimensional spin-1/2 Ising model in the presence of an external constant magnetic field with arbitrary orientation is

$$
\mathbf{H}=\sum_{i=1}^{N}\left(J S_{i}^{z} S_{i+1}^{z}-h_{y} S_{i}^{y}-h_{z} S_{i}^{z}\right)
$$

where $S_{i}^{y}=\sigma_{y} / 2$ and $S_{i}^{z}=\sigma_{z} / 2$, where $\sigma_{y}$ and $\sigma_{z}$ are the Pauli matrices. The coupling constant $J$ can be either positive (antiferromagnetic model) or negative (ferromagnetic model). The chain has $N$ spatial sites and it satisfies periodic spatial boundary conditions. Due to the symmetry of the Hamiltonian along the $z$-direction (the easy-axis of the chain), the most general constant external magnetic field that we must consider can be written as $\mathbf{h}=h_{y} \hat{j}+h_{z} \hat{k}$, where the components $h_{y}$ and $h_{z}$ are positive constants.

The method of Ref. [9] has been applied directly to the Hamiltonian (1) to yield the high temperature expansion of its HFE, in the thermodynamical limit $(N \rightarrow \infty)$, up to order $\beta^{7}$. This expansion is presented in appendix (A).

From the expansion (A.1) we verify that: $i$ ) the HFE (A.1) is an even function of the $y$ and $z$ components of the external magnetic field; $i i$ ) the HFE is sensitive to the sign of $J$ (thus distinguishing between the ferromagnetic and antiferromagnetic cases) only for non-zero values of $h_{z}$. Even for $h_{z}=0$ there are thermodynamical functions that can distinguish one model from the other (e.g., the first-neighbour $z$-component spin correlation function and the $z z$-component of the magnetic susceptibility tensor).

Substituting $h_{y}=0$ or $h_{z}=0$ in the expansion (A.1) recovers the thermodynamics of the corresponding limiting cases of the Hamiltonian (1), given in Refs. [2] and [3], respectively.

\section{THERMODYNAMICAL FUNCTIONS AT HIGH TEMPERATURES}

One can obtain the exact HFE of the one-dimensional spin$1 / 2$ Ising model for two specific configurations of the external magnetic field; namely, the longitudinal[2] and transverse [3] ones. But that information, by itself, is not enough to infer the thermodynamic behavior for intermediary configurations of the magnetic field.

Our aim is to compare these thermodynamical functions for the ferro- and antiferromagnetic models, keeping the norm of the external magnetic field fixed, but letting its spatial orientation vary; i.e., by considering a magnetic field of the form

$$
\mathbf{h}=h \sin (\theta) \hat{j}+h \cos (\theta) \hat{k},
$$

where $h=|\mathbf{h}|$ is the constant norm and $\theta \in[0, \pi / 2]$ is the angle between $\mathbf{h}$ and the $z$-axis.

\section{A. Crossing the critical line at $T=0 \mathrm{~K}$}

The spin-1/2 Hamiltonian (1) has been studied at $T=0 K$ for the ferromagnetic[6] $(J /|J|=-1)$ and the antiferromagnetic[7] $(J /|J|=1)$ cases. The ground-state phase diagram [7] of the antiferromagnetic model in the presence of a skew magnetic field has a critical line that separates the antiferromagnetic and paramagnetic states.

It is a well-known (exact) result that the spin- $1 / 2$ Ising model has no phase transition at finite temperature, for a purely longitudinal or transversal magnetic field. It is certainly interesting to verify explicitly the behaviour of the Ising model in a skew magnetic field at finite temperature, when the critical line at $T=0 \mathrm{~K}$ is crossed. We do so in this subsection by choosing $|J| \beta=0.7$ (which is not such a high temperature) and $h /|J|=0.53$, since for the $T=0 \mathrm{~K}$ antiferromagnetic $(J /|J|=1)$ case the critical line in the phase diagram is 

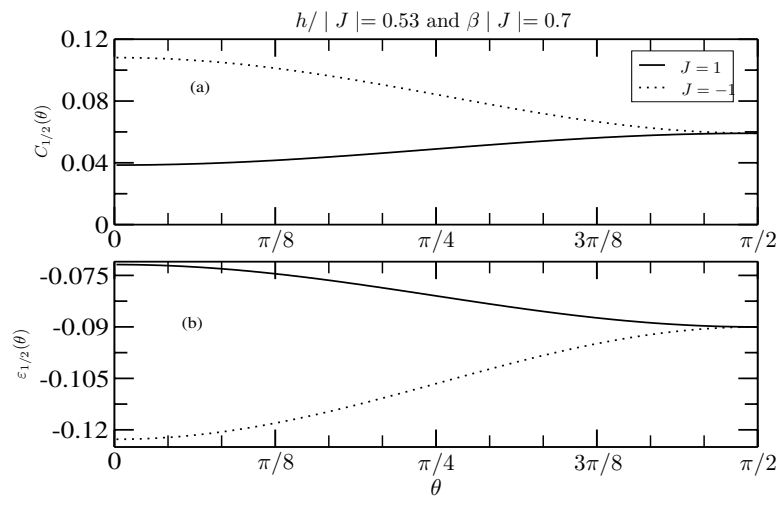

FIG. 1: (a) The specific heat per site; (b) the internal energy per site. The curves are plotted as functions of $\theta$, the angle between the external magnetic field vector and the easy-axis. The norm of the magnetic field, in units of $|J|$, is kept constant as 0.53 . The angle $\theta$ varies in the interval $[0, \pi / 2]$ and $|J| \beta=0.7$.

indeed crossed, for $\theta \in[0, \pi / 2]$. Each plot that follows compares the behaviour of ferromagnetic and antiferromagnetic cases under rotation of the magnetic field.

In the following figures we use the convention that the dotted (or dashed) and solid lines correspond to the ferromagnetic and to the antiferromagnetic model, respectively.

We begin the discussion of the thermodynamical functions of this model by the specific heat per site $C_{1 / 2}(\beta) \equiv$ $-\beta^{2} \frac{\partial^{2}\left(\beta \mathcal{W}_{1 / 2}\right)}{\partial \beta^{2}}$, plotted in Fig.(1a) as a function of $\theta$. At $\theta=0$ (longitudinal magnetic field) and $\theta=\pi / 2$ (transverse magnetic field) we have $\frac{\mathrm{d} C_{1 / 2}}{\mathrm{~d} \theta}=0$. The second derivative of this thermodynamical function is different at those two values of $\theta$ for the two models, but for each of them there is a single value $\bar{\theta}$, that depends weakly on the value of $\beta$ and on the model, where $\left.\frac{d^{2} C_{1 / 2}}{d \theta^{2}}\right|_{\theta=\bar{\theta}}=0$. The curve of the specific heat per site is a monotonically decreasing function of $\theta$ for the antiferromagnetic model, whereas it is a monotonically increasing function for the ferromagnetic model in the same interval of $\theta$ for $|J| \beta \lesssim 1$. For each curve, the concavity changes only once; the value of $\bar{\theta}$ is slightly different for the two models.

The internal energy per site $\varepsilon_{1 / 2}(\beta) \equiv \frac{\partial\left(\beta \mathcal{W}_{1 / 2}\right)}{\partial \beta}$ is plotted in Fig. (1b). At $\theta=0$ and $\pi / 2$ we have $\frac{d \varepsilon_{1 / 2}}{d \theta}=0$ for both models. The concavity of each curve changes at a single value of $\bar{\theta}$ which depends weakly on the value of $|J| \beta$, and is slightly different for the two models. For $|J| \beta \lesssim 1$, the internal energy of the antiferromagnetic model is a monotonically decreasing function of $\theta$ whereas for the ferromagnetic model it is monotonically increasing.

The ferromagnetic and antiferromagnetic cases for the two thermodynamical functions in Fig. (1) coincide at $\theta=\pi / 2$ (transversal magnetic field) since the HFE of the Hamiltonian (1) with a transverse magnetic field $\left(h_{z}=0\right)$ is insensitive to the sign of $J$.

Fig. (2a) shows the first-neighbour z-component spin cor-

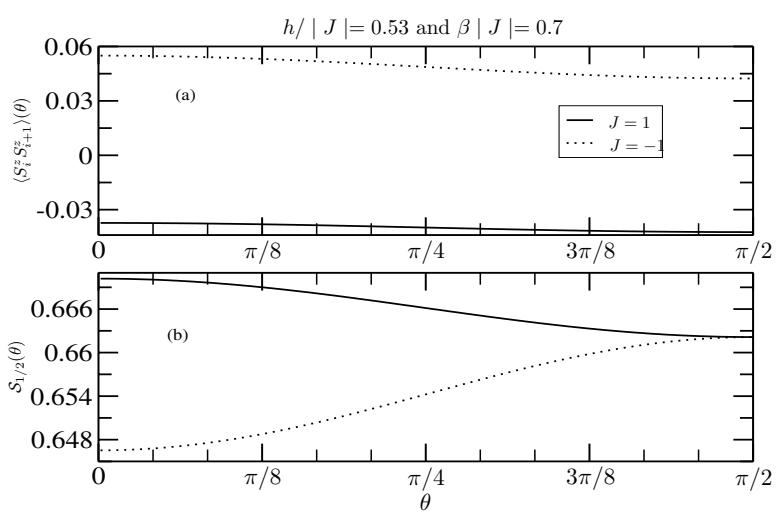

FIG. 2: (a) The first-neighbour $z$-component of spin correlation function. (b) The entropy for the ferromagnetic $(J /|J|=-1)$ and antiferromagnetic $(J /|J|=1)$ models under the same conditions as those of Fig. (1). Both thermodynamical functions are plotted as functions of $\theta \in[0, \pi / 2]$.

relation function $\left\langle S_{i}^{z} S_{i+1}^{z}\right\rangle(\beta) \equiv \frac{\partial \mathcal{W}_{1 / 2}}{\partial J}$ for $J /|J|=1$ (antiferromagnetic case) and $J /|J|=-1$ (ferromagnetic case). For both curves we have $\mathrm{d}\left\langle S_{i}^{z} S_{i+1}^{z}\right\rangle / \mathrm{d} \theta=0$ at $\theta=0$ and $\pi / 2$. As it should be, this correlation function decreases for the two models as $\theta$ increases. The concavity of the curves changes for a single value of $\bar{\theta}$, that depends weakly on $|J| \beta$ and on the model. Only for $\theta=\pi / 2$, we have that $\left.\left\langle S_{i}^{z} S_{i+1}^{z}\right\rangle\right|_{J /|J|=1}=$ $-\left.\left\langle S_{i}^{z} S_{i+1}^{z}\right\rangle\right|_{J / J \mid=-1}$, at least in the region $|J| \beta \lesssim 1$ and for $h /|J| \lesssim 0.7$.

The entropy per site $S_{1 / 2}(\beta) \equiv \beta^{2} \frac{\partial \mathcal{W}_{1 / 2}}{\partial \beta}$ for the ferromagnetic and antiferromagnetic models is plotted in Fig. (2b). The first derivative of the function $S_{1 / 2}$ with respect to $\theta$ is zero at $\theta=0$ and $\pi / 2$. This thermodynamical function at $\theta=\pi / 2$ is also the same for $J /|J|= \pm 1$, because, at this orientation of the magnetic field, the HFE is an even function of $J$. Again, the concavity of the curves, for both models, changes for a single value $\bar{\theta}$ that depends weakly on $|J| \beta$ and on the model.

The $y$ and $z$ components of the magnetization per site, $\mathcal{M}_{y}^{(1 / 2)}(\beta) \equiv-\frac{\partial \mathcal{W}_{1 / 2}}{\partial h_{y}}$ and $\mathcal{M}_{z}^{(1 / 2)}(\beta) \equiv-\frac{\partial \mathcal{W}_{1 / 2}}{\partial h_{z}}$ are presented in Figs. (3). Fig. (3a) shows that $\mathcal{M}_{y}^{(1 / 2)}(\theta)$ is almost the same for both models, at least for $|J| \beta \lesssim 1$ and $h /|J| \lesssim 0.7$. The percentual difference between them is smaller than $3 \%$ for $\theta \in[0, \pi / 2]$ and the largest difference occurs around $\theta=0$. This difference decreases as the value of $|J| \beta$ decreases. For $\theta \sim 0$, we have $\mathcal{M}_{y}^{(1 / 2)}(\theta) \approx a \theta$, and the coefficient $a$ depends on $\beta$. At $\theta=\pi / 2$ we have $\mathrm{d} \mathcal{M}_{y}^{(1 / 2)}(\theta) / \mathrm{d} \theta=0$, for $J /|J|= \pm 1$.

On the other hand, $\mathcal{M}_{z}^{(1 / 2)}$ differs for the two cases (see Fig. (3b)), except at $\theta=\pi / 2$. Around $\theta=\pi / 2, \mathcal{M}_{z}^{(1 / 2)}$ is a linear function of $\theta: \mathcal{M}_{z}^{(1 / 2)} \approx b(\pi / 2-\theta)$. The coefficient $b$ depends on the value of $\beta$ and on the model. We also have $d \mathcal{M}_{z}^{(1 / 2)}(\theta) / d \theta=0$ at $\theta=0$.

The last thermodynamical function to be discussed is the magnetic susceptibility tensor $\chi_{i j}^{(1 / 2)}(\beta) \equiv-\frac{\partial^{2} \mathcal{W}_{1 / 2}}{\partial h_{i} \partial h_{j}}$, where 

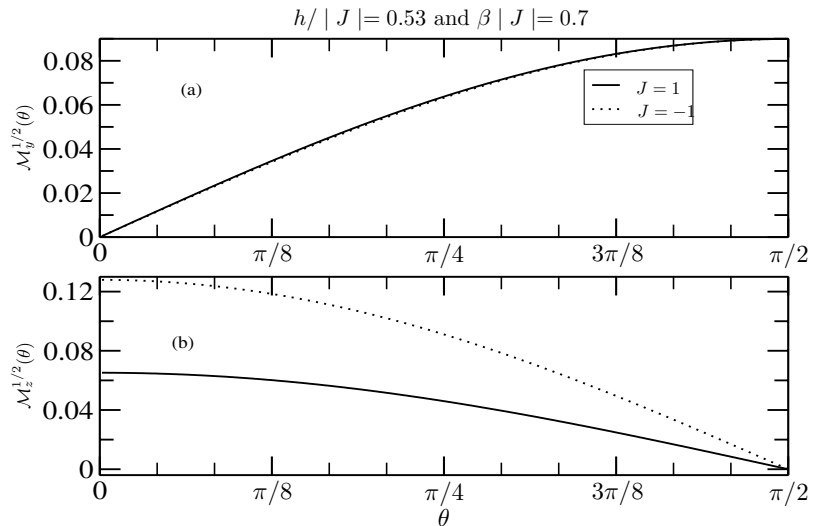

FIG. 3: The $\mathcal{M}_{y}^{1 / 2}$ and $\mathcal{M}_{z}^{1 / 2}$ components of the magnetization per site, in (a) and (b), respectively, as functions of the angle $\theta$ between the vector magnetic field and the $z$-axis. Here, $|J| \beta=0.7$ and $h /|J|=$ 0.53 .

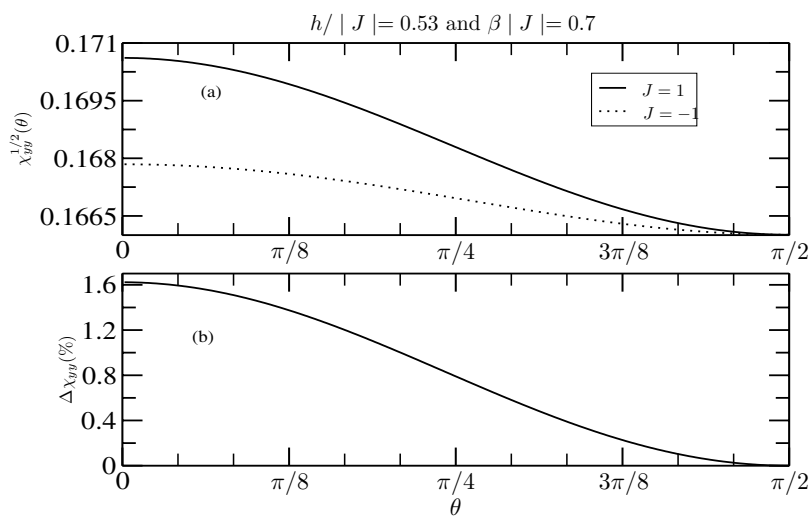

FIG. 4: (a) The element $\chi_{y y}^{1 / 2}$ of the magnetic susceptibility tensor versus $\theta$, for the antiferromagnetic and ferromagnetic models, under the same conditions as the previous figures. (b) The corresponding percentual difference of $\chi_{y y}^{1 / 2}$, comparing the ferro- and antiferromagnetic models.

$i, j \in\{y, z\}$. Fig. (4a) shows the component $\chi_{y y}^{(1 / 2)}$ as a function of $\theta$; even at $|J| \beta=0.7$, this function for the ferromagnetic model (dashed line) is almost constant for $\theta \in[0, \pi / 2]$. The percentual difference of the two curves is shown in Fig. (4b); the two models have a similar value of $\chi_{y y}^{(1 / 2)}$ for any direction of the external magnetic field. At $|J| \beta=0.7$, the largest percentual difference of this element of the magnetic susceptibility tensor is $3 \%$ for the two models for $h /|J| \lesssim 0.7$. At $\theta=0$ we have $\chi_{y y}^{(1 / 2)} \neq 0$, for $J /|J|= \pm 1$. This last result cannot be derived from the HFE of the model known in the literature[2]. The non-zero contributions to $\chi_{y y}^{(1 / 2)}(0)$ come from terms in the expansion (A.1) of the type $J^{2 p} h_{y}^{2}$, where $p=0,1,2$ and 3 . For $J /|J|= \pm 1$, we also have $\mathrm{d} \chi_{y y}^{(1 / 2)} / \mathrm{d} \theta=0$ at $\theta=0$ and $\pi / 2$, and at $\theta=\pi / 2$ the component $\chi_{y y}^{(1 / 2)}$ is the same for the
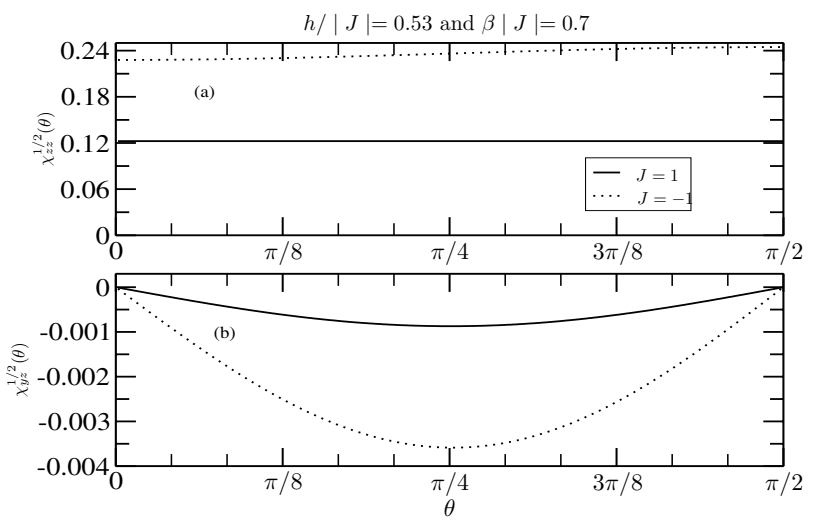

FIG. 5: (a) $\chi_{z z}^{1 / 2}$ versus $\theta$ for the antiferromagnetic $(J /|J|=1)$ and ferromagnetic $(J /|J|=-1)$ models, with $|J| \beta=0.7$ and $h /|J|=$ 0.53. (b) $\chi_{y z}^{1 / 2}$, under the same conditions.

ferro- and antiferromagnetic models.

Fig. (5a) shows the $z z$-component of the magnetic susceptibility tensor of the two models. The interesting point about this graph is that at $\theta=\pi / 2$ the value of the $\chi_{z z}^{(1 / 2)}$ is nonzero and is different for $J /|J|= \pm 1$. We point out that, in both ferro- and antiferromagnetic cases, the expansion (A.1) shows that the rotation of the magnetic field up to the $\theta=\pi / 2$ configuration (i.e., a purely transverse magnetic field) yields a non-vanishing $\chi_{z z}^{(1 / 2)}$. This result cannot be derived from the exact HFE of Ref. [3]. For the ferro- and antiferromagnetic models in the presence of a mixed magnetic field we have $\mathrm{d} \chi_{z z}^{(1 / 2)}(\theta) / \mathrm{d} \theta=0$ at $\theta=0$ and $\pi / 2$. The function $\chi_{z z}^{(1 / 2)}(\theta)$ for the antiferromagnetic model is almost constant for $\theta \in[0, \pi / 2]$ for $|J| \beta \lesssim 1$ and $h /|J| \lesssim 0.6$, in which our $\beta$-expansion is sound. The non-null contributions to $\chi_{z z}^{(1 / 2)}(\theta)$ that give different contributions for the ferro- and antiferromagnetic models come from the terms in the $\beta$-expansion (A.1) of the type $J^{p} h_{z}^{2}$, where $p=0,1, \cdots, 6$.

Fig. (5b) shows $\chi_{y z}^{(1 / 2)}(\theta)$ for the two models; although it is not zero, from Figs. (5) we verify that the non-diagonal elements of the magnetic susceptibility tensor are much smaller than the diagonal elements, for both models. We also have $\chi_{y z}^{(1 / 2)}(0)=\chi_{y z}^{(1 / 2)}(\pi / 2)=0$ for the ferro- and antiferromagnetic models. We are able to calculate all the elements of the tensor $\chi_{i j}^{(1 / 2)}(\beta)$ only if we have the Ising model (1) in the presence of a skew magnetic field.

In Figs. (1)-(5) we maintain $|J| \beta=0.7$ and $h /|J|=0.53$, although the features described previously in the curves of the ferromagnetic and the antiferromagnetic models, such as the monotonically increasing/decreasing behaviour of the functions for $\theta \in[0, \pi / 2]$ and vanishing first derivatives at $\theta=0$ and/or $\pi / 2$, are preserved, at least for $|J| \beta \lesssim 1$ and $h /|J| \lesssim 0.7$, for which our expansion (A.1) is bona fide. 

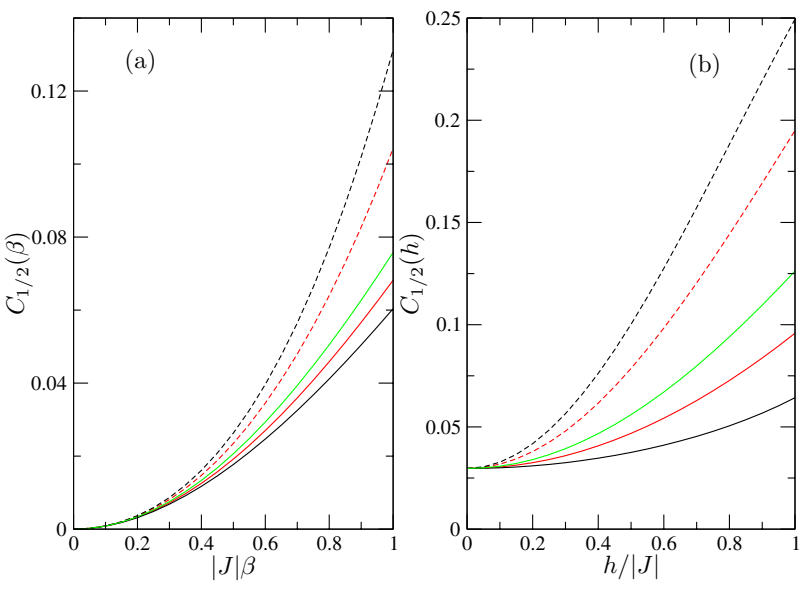

FIG. 6: The specific heat per site at $\theta=0$ (black curves), $\pi / 4$ (red curves) and $\pi / 2$ (green curves) for the ferro- and antiferromagnetic models. In (a) the specific heat is plotted versus $|J| \beta$ at $h /|J|=0.3$; and in (b), as a function of $h /|J|$ at $|J| \beta=0.7$.

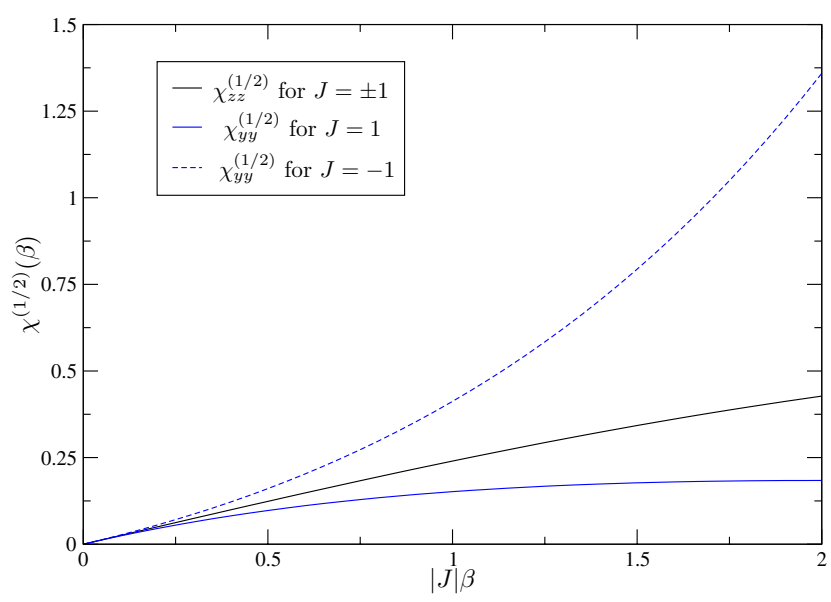

FIG. 7: The diagonal components of the static magnetic susceptibility tensor as functions of $|J| \beta$. The component $\chi_{y y}^{(1 / 2)}$ corresponds to the black curve and $\chi_{z z}^{(1 / 2)}$ to the blue curve.
B. Other aspects of the thermodynamics in the region $|J| \beta \lesssim 1$

It is certainly interesting to check the behavior of the thermodynamics of the model described by the Hamiltonian (1) as a function of the temperature, (or of its inverse), and its dependence on the external magnetic field.

Figs. (6a) and Fig.(6b) show the specific heat per site as a function of $|J| \beta$ and $h /|J|$, respectively, at $\theta=0, \pi / 4$ and $\pi / 2$. From those figures, some aspects of the specific heat can be inferred that Fig.(1a) would not allow.

Fig.(6a) shows that the specific heat per site for $|J| \beta \lesssim 0.2$ and for all values of $\theta \in[0, \pi / 2]$ is independent of the model. This is easily understandable if we observe the $\beta$-expansion of this thermodynamical function, derived from the HFE (A.1). From this series we obtain that the spin-1/2 Ising model in the presence of a skew magnetic field presents a tail of the Schottky peak[10] $\left(C_{S c h} \propto \beta^{2}\right)$, for all values of $\theta$. The coefficient of the $\beta^{2}$-term is independent of the sign of $J$. In Fig.(6a) we take $h /|J|=0.3$. The black curves in this figure present the specific heat of the ferro- and antiferromagnetic models at $\theta=0$; the red curves correspond these functions at $\theta=\pi / 4$ and the green curve corresponds to the specific heat at $\theta=\pi / 2$, which is the same for both models.

Fig.(6b) shows the specific heat per site as a function of $h /|J|$, at $|J| \beta=0.7$, for $\theta=0, \pi / 4$ and $\pi / 2$. At $h=0$, the specific heat is non-null and its value is independent of the sign of $J$. The black and red curves describe the specific heat per site at $\theta=0$ and $\theta=\pi / 4$, respectively, for the ferro- and antiferromagnetic models, and the green curve corresponds to this function at $\theta=\pi / 2$, which is the same for both models. We point out that, in contrast to the expansion in $|J| \beta$, the expansion in terms of the norm $h /|J|$ of the magnetic field is not exact, i.e., each coefficient in this expansion has corrections from higher orders in $\beta$. Up to order $\beta^{7}$, we obtain that the specific heat for a vanishing magnetic field is

$$
\left.C_{1 / 2}(\beta)\right|_{h=0}=-\frac{17}{2949120} \beta^{8} J^{8}+\frac{1}{6144} \beta^{6} J^{6}-\frac{1}{256} \beta^{4} J^{4}+\frac{1}{16} \beta^{2} J^{2}
$$

The static magnetic susceptibility tensor at $h=0$ has only nonnull diagonal elements. None of those elements can be derived from the exact HFE of the longitudinal[1] or the transversal[3] Ising models. Fig.(7) shows the $y y$-component of the static magnetic susceptibility tensor $\left(\chi_{y y}^{(1 / 2)}\right)$ (black curve), which is the same for both models, and the $z z$-component $\left(\chi_{z z}^{(1 / 2)}\right)$ of the ferro- (blue dashed line) and the antiferromagnetic (blue continuous line) model. In the region $|J| \beta \lesssim 0.3$, the elements $\chi_{y y}^{(1 / 2)}$ and $\chi_{z z}^{(1 / 2)}$ of both models are very close. For $h=0$, our $\beta$-expansion of the elements of this tensor are reliable up to 

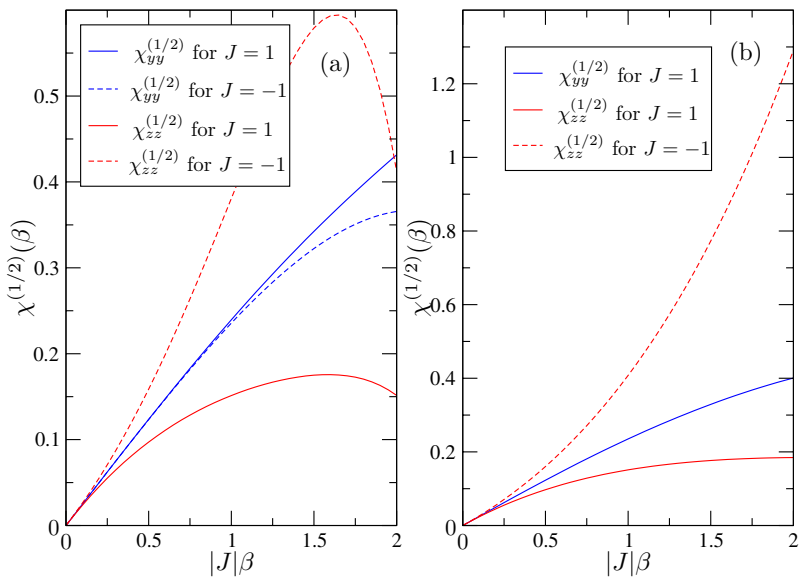

FIG. 8: The components $\chi_{y y}^{(1 / 2)}$ (blue curves) and $\chi_{z z}^{(1 / 2)}$ (red curves) for the purely longitudinal and transversal magnetic field configurations, for $h /|J|=0.3$. In (a) we have the longitudinal external magnetic field, parallel to the easy-axis $(\theta=0)$; and in (b) the transversal magnetic field, perpendicular to the easy-axis $(\theta=\pi / 2)$.

\section{$|J| \beta \sim 2$.}

The component $\chi_{y y}^{(1 / 2)}$ for the purely longitudinal $(\theta=0)$ model is non-null. It cannot be derived from the HFE of the exact model[1]. Fig.(8a) shows $\chi_{y y}^{(1 / 2)}$ and $\chi_{z z}^{(1 / 2)}$ for $h /|J|=$ 0.3 and $\theta=0$ (purely longitudinal external field). The blue curves represent $\chi_{y y}^{(1 / 2)}$ of the ferro- and the antiferromagnetic cases. The element $\chi_{y y}^{(1 / 2)}$ of both models have very close values, up to $|J| \beta \sim 1$. There is a peak of the ferromagnetic $\chi_{z z}^{(1 / 2)}$ at $|J| \beta=1.6342$.

The component $\chi_{z z}^{(1 / 2)}$ of the purely transversal $(\theta=\pi / 2)$ case is also non-null. Fig.(8b) shows the diagonal components $\chi_{y y}^{(1 / 2)}$ and $\chi_{z z}^{(1 / 2)}$ for $h /|J|=0.3$ and $\theta=\pi / 2$. The blue curve corresponds to $\chi_{y y}^{(1 / 2)}$, which is the same for both models; the red curves describe $\chi_{z z}^{(1 / 2)}$ for the ferro- and antiferromagnetic models.

For the purely longitudinal/transversal configurations of the magnetic field, the elements $\chi_{y y}^{(1 / 2)}$ and $\chi_{z z}^{(1 / 2)}$ are of the same order of magnitude.

The one-dimensional spin-1/2XY model,

$$
\mathbf{H}_{X Y}=\sum_{i=1}^{N}\left(J_{x} S_{i}^{x} S_{i+1}^{x}+J_{y} S_{i}^{y} S_{i+1}^{y}\right)
$$

(where $N$, the number of sites, is even) is equivalent to two spin-1/2 Ising models with transversal magnetic field (Hamiltonian (1) with $h_{z}=0$ ). This equivalence was demonstrated [11] and used in the late 70's and early 80's to infer quantum properties from one model based on the other $[12,13]$. The lattice spacing of the two Ising models is twice as large as that of the $X Y$ model.
One interesting fact also to be verified is the equivalence of the spin-1/2XY model, whose Hamiltonian is

$$
\mathbf{H}_{X Y}=\sum_{i=1}^{N}\left(J S_{i}^{x} S_{i+1}^{x} \pm 2 h_{y} S_{i}^{y} S_{i+1}^{y}\right),
$$

to the spin-1/2 Ising model in the presence of a transversal magnetic field (Hamiltonian (1) with $h_{z}=0$ ) at finite temperature. In Ref. [14] we present the HFE of the spin- $S X Y Z$ model for an external magnetic field and with a single-ion anisotropy term, up to order $\beta^{5}$. This new equivalence can be verified directly by comparing the HFE (A.1) for the spin$1 / 2$ Ising model with transversal magnetic field $\left(h_{z}=0\right)$ to the high temperature expansion presented in Ref. [14] for the $X Y$ chain $\left(J_{z}=0\right)$. These HFE are calculated taking the thermodynamical limit of the models; the number of sites does not need to be even, as imposed in Ref. [11]. This new equivalence could also be shown by the equality of the dispersion relations of the two models[5, 6].

Upon finishing the study of the thermodynamics of Hamiltonian (1), we would like to comment on the statement in Ref. [6] where it is claimed that at $T=0 \mathrm{~K}$ the spin- $1 / 2$ Ising model with a skew external magnetic field (1) is "dynamically quite similar" to the special case of the $X Y Z$ magnetic chain, that is

$$
\mathbf{H}^{\prime}=\sum_{i=1}^{N}\left(J_{x}\left(S_{i}^{x} S_{i+1}^{x}-S_{i}^{y} S_{i+1}^{y}\right)+J S_{i}^{z} S_{i+1}^{z}-h_{z} S_{i}^{z}\right) .
$$

Ref. [6] suggests the existence of some relation (although unspecified therein) between the magnetic field component $h_{y}$ of the Hamiltonian (1) and the exchange parameter $J_{x}$ of Eq. (6), at $T=0 \mathrm{~K}$. In order to verify whether this would still hold for finite temperatures, we compared the thermodynamical functions calculated here to their results. From the $\beta$-expansion of the HFE for the $X Y Z$ chain model [14], we verify that it depends only on even powers of $J_{x}$ and $J_{y}$; consequently, the Hamiltonian (6) also describes an $X X Z$ chain in the presence of an external magnetic field along the $z$-direction. In Ref. [15] we present the high temperature expansion of the spin- $S$ $X X Z$ model, with a single-ion anisotropy term and in the presence of a longitudinal magnetic field $\left(h_{y}=0\right.$ and $h_{z} \neq 0$ ), up to order $\beta^{6}$.

We assume a linear relation between $J_{x}$ and $h_{y}$, independent of the value of $\beta$; that is, $h_{y}=a J_{x}$. In Figs. (9) we have $J=1$, $J_{x}=1.5$ and $h_{z}=0.5$. Fig. (9a) shows the percentual difference between the HFE of the Ising model (1) and that of the spin-1/2 XXZ model (6). This curve corresponds to the minimum difference between the curves representing the HFEs of those models, and it is obtained by choosing $a=0.7562$ for $\beta \in[0,0.9]$. The maximum percentual difference between the two HFEs is $0.18 \%$ for the interval of $\beta$ shown in this figure. In Fig. (9b) we plot the percentual difference between the two models for the following thermodynamical functions per site: the specific heat, the mean energy, the entropy, the first-neighbour $z$-component of the spin correlation function and the $z$-component of the magnetization, for the same set 

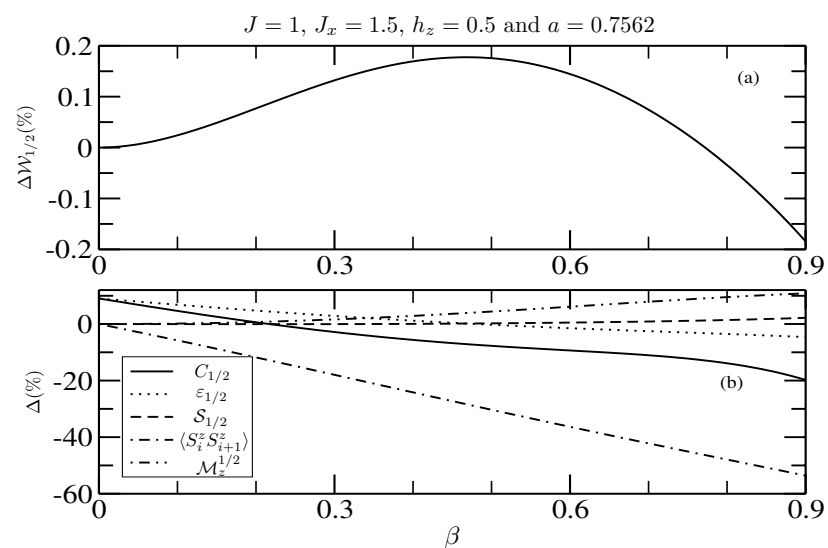

FIG. 9: (a) The percentual difference of the HFE for the spin- $1 / 2$ models: the Ising model with a skew magnetic field and the XXZ chain in the presence of a magnetic field in the $z$-direction. (b) The percentual difference of the specific heat, the first-neighbour $S_{z}$ correlation, the entropy, the internal energy and the magnetization. Here, we let $J=1, J_{x}=1.5, h_{z}=0.5$ and $a=0.7562$.

of constants as Fig. (9a). From these curves, we verify that the entropy of the two models is similar, since the maximum percentual difference in this interval of $\beta$ is $1.51 \%$. On the other hand, the remaining functions are very distinct; for instance, the first-neighbor $S_{z}$ correlation and the specific heat reach percentual differences of $53.3 \%$ and $19.35 \%$, respectively, at $\beta=0.9$. From Fig. (9b) we can state that the thermodynamics of Hamiltonians (1) and (6) are not similar, at least in the region of $|J| \beta \lesssim 1$.

\section{CONCLUSIONS}

We derive the thermodynamics of the one-dimensional spin-1/2 Ising model (ferromagnetic and antiferromagnetic cases) in the presence of an arbitrary external constant magnetic field, in the high temperature region $(|J| \beta \lesssim 1)$. The $\beta$-expansion of the HFE of the model is calculated up to or$\operatorname{der} \beta^{7}$. From expansion (A.1) we recover the known limiting cases of the model with $h_{y}=0$ (longitudinal magnetic field)[2] or $h_{z}=0$ (transversal magnetic field) [3].

In the present communication, the magnetic field vector is supposed to have a constant norm and is rotated with respect to the easy-axis ( $z$-direction) by an angle $\theta$, which varies in the interval $[0, \pi / 2]$. The phase diagram of the spin- $1 / 2$ antiferromagnetic Ising model with skew magnetic field exhibits a critical line, in the space of magnetic field components $\left(h_{y}, h_{z}\right)$ at zero temperature[7]. In the first part of the paper the norm of the magnetic field has been chosen to be $h /|J|=0.53$, such that there exists a critical angle $\theta_{c}$ for which the zerotemperature critical line of the phase diagram is crossed, as $\theta$ varies. The following thermodynamical functions per site are plotted as functions of $\theta$, for the antiferromagnetic and the ferromagnetic models at $|J| \beta=0.7$ : the specific heat; the in- ternal energy; the first-neighbour $S_{z}$ correlation function; the entropy; the $y$ and $z$ components of the magnetization; and the elements of the magnetic susceptibility tensor. All these are smooth functions of $\theta$ in the interval $[0, \pi / 2]$. Although the curves are plotted for $|J| \beta=0.7$ and $h /|J|=0.53$, the features presented and discussed in the text are preserved, at least, up to $|J| \beta \sim 1$ and $h /|J| \sim 0.7$. In general, the curves are monotonically increasing or decreasing functions of $\theta$.

The $y$-component of the magnetization and the magnetic susceptibility component $\chi_{y y}^{(1 / 2)}$ of antiferro- and ferromagnetic models are very close. The component $\chi_{z z}^{(1 / 2)}$ for the ferromagnetic case is almost independent of the $\theta$ angle. Those results are valid, at least, for $|J| \beta \lesssim 1$ and $h /|J| \lesssim 0.7$.

Our results for the elements $\chi_{y y}^{(1 / 2)}$ and $\chi_{z z}^{(1 / 2)}$ of the magnetic susceptibility tensor for the limiting cases $h_{y}=0$ and $h_{z}=0$, respectively, could not be derived from the known HFE in the literature[2,3]. These elements are non-zero.

We verify that the spin-1/2 Ising model in a skew magnetic field presents a tail of the Schottky peak[10] that is $\theta$ dependent, but otherwise independent of other features of the model. We also plot the specific heat per site versus the norm of the magnetic field. This function does not vanish at $h=0$, and gets corrections from terms of higher orders in $\beta$ from high temperature expansion of the HFE of the model.

By explicit calculation, we verify the equivalence of the spin-1/2 Ising model in the presence of a transversal magnetic field and the $X Y$ chain, a different duality of the one known in the literature[3, 11, 13].

Finally, we verified that the thermodynamics of the spin$1 / 2$ Ising model with a skew magnetic field is not similar to the thermodynamics of the spin- $1 / 2 X X Z$ chain, at least in this region of temperature, contrary to what is claimed by Fogedby[6] to hold for $T=0 \mathrm{~K}$.

S.M. de S. and O.R. thank FAPEMIG and CNPq for partial financial support. E.V. Corrêa Silva (Fellowship CNPq, Brasil, Proc.No.305800/2004-3) thanks CNPq for partial financial support (Edital Universal CNPq/2006 Proc.No.476852/2006-4). M.T. Thomaz (Fellowship CNPq, Brasil, Proc.No.: 30.0549/83-FA) thanks CNPq for partial financial support (Grants 1D). J.E.F. Skea thanks FAPERJ for financial support via a Prociência research grant during part of this work.

\section{APPENDIX A: THE $\beta$-EXPANSION OF THE HFE FOR THE SPIN-1/2 ISING MODEL WITH SKEW MAGNETIC FIELD}

Hamiltonian (1) describes the interaction between first neighbours along the chain, subject to a periodic spatial condition. These two features allow us to apply the method of Ref. [9] directly to this Hamiltonian to calculate the $\beta$ expansion of its HFE.

The HFE of the spin-1/2 Ising model in the presence of a mixed external constant magnetic field, up to order $\beta^{7}$, is 


$$
\begin{aligned}
\mathcal{W}_{1 / 2}\left(\beta ; h_{y}, h_{z}\right) & =\left(\frac{17}{645120} h_{z}{ }^{8}-\frac{107}{35840} J^{2} h_{y}{ }^{2} h_{z}{ }^{4}+\frac{17}{161280} h_{z}{ }^{6} h_{y}{ }^{2}-\frac{1}{1792} J^{2} h_{z}{ }^{2} h_{y}{ }^{4}\right. \\
& +\frac{17}{645120} h_{y}{ }^{2}+\frac{17}{161280} h_{z}{ }^{2} h_{y}{ }^{6}+\frac{17}{107520} h_{z}{ }^{4} h_{y}{ }^{4}+\frac{17}{2580480} J^{6} h_{y}{ }^{2} \\
& +\frac{121}{73728} J^{4} h_{z}{ }^{4}+\frac{17}{165150720} J^{8}+\frac{61}{1290240} J^{4} h_{y}{ }^{2} h_{z}{ }^{2}-\frac{107}{46080} J^{2} h_{z}{ }^{6} \\
& \left.+\frac{17}{161280} J^{2} h_{y}{ }^{6}+\frac{17}{286720} J^{4} h_{y}{ }^{4}-\frac{1}{368640} J^{6} h_{z}{ }^{2}\right) \beta^{7}+\left(\frac{17}{11520} J h_{z}{ }^{6}\right. \\
& +\frac{17}{11520} J h_{z}{ }^{2} h_{y}{ }^{4}+\frac{17}{5760} J h_{z}{ }^{4} h_{y}{ }^{2}-\frac{1}{1920} J^{3} h_{z}{ }^{2} h_{y}{ }^{2}-\frac{5}{1152} J^{3} h_{z}{ }^{4} \\
& \left.+\frac{1}{30720} J^{5} h_{z}{ }^{2}\right) \beta^{6}+\left(\frac{13}{3840} J^{2} h_{z}{ }^{2} h_{y}{ }^{2}-\frac{1}{2880} h_{y}{ }^{6}-\frac{1}{184320} J^{6}-\frac{1}{2880} h_{z}{ }^{6}\right. \\
& -\frac{1}{960} h_{z}{ }^{4} h_{y}{ }^{2}-\frac{1}{960} h_{z}{ }^{2} h_{y}{ }^{4}-\frac{1}{5120} J^{4} h_{y}{ }^{2}-\frac{1}{3072} J^{4} h_{z}{ }^{2}+\frac{13}{1536} J^{2} h_{z}{ }^{4} \\
& \left.-\frac{1}{1280} J^{2} h_{y}{ }^{4}\right) \beta^{5}+\left(\frac{1}{384} J^{3} h_{z}{ }^{2}-\frac{1}{96} J h_{z}{ }^{2} h_{y}{ }^{2}-\frac{1}{96} J h_{z}{ }^{4}\right) \beta^{4} \\
& +\left(-\frac{1}{64} J^{2} h_{z}{ }^{2}+\frac{1}{3072} J^{4}+\frac{1}{96} h_{z}{ }^{2} h_{y}{ }^{2}+\frac{1}{192} h_{y}{ }^{4}+\frac{1}{192} h_{z}{ }^{4}\right. \\
& \left.+\frac{1}{192} J^{2} h_{y}{ }^{2}\right) \beta^{3}+\frac{\beta^{2} J h_{z}{ }^{2}}{16}+\left(-\frac{h_{z}{ }^{2}}{8}-\frac{J^{2}}{32}-\frac{h_{y}{ }^{2}}{8}\right) \beta-\frac{\ln (2)}{\beta} .
\end{aligned}
$$

The function $\mathcal{W}_{1 / 2}\left(\beta ; h_{y}, h_{z}\right)$ is sensitive to the sign of $J$ only if $h_{z} \neq 0$. The expansion (A.1) is equally valid for positive (antiferromagnetic case) and negative (ferromagnetic case) values of $J$.
Certainly, the proper parameter in which one could rewrite (A.1) is $|J| \beta$; therefore, the components of the external magnetic field are measured in units of $|J|$.
[1] E. Ising, Z. Phys. 31, 253 (1925).

[2] H.A. Kramers and G.H. Wannier, Phys. Rev. 60, 252 (1941).

[3] P. Pfeuty, Annals of Phys. 57, 79 (1970).

[4] A. Kopp and S. Chakravarty, Nature 1, 53, October (2005).

[5] M. Takahashi, "Thermodynamic of One-Dimensional Solvable Models", (Cambridge University Press, Cambridge, 1999).

[6] H.C. Fogedby, J. Phys. C 11, 2801 (1978).

[7] A.A. Ovchinnikov, D.V. Dmitriev, V. Ya. Krivnov, and V.O. Cheranovskii, Phys. Rev. B 68, 214406 (2003).

[8] R. Clérac, H. Miyasaka, M. Yamashita, and C. Coulon, J. Am. Chem. Soc 124, 12837 (2002).

[9] O. Rojas, S. M. de Souza, and M. T. Thomaz, J. Math. Phys.

\section{3, 1390 (2002).}

[10] S.J. Collocott, R. Driver, L. Dale, and S.X. Dou, Phys. Rev. B 37, 7917 (1988).

[11] R. Jullien and J.N. Fields, Phys. Lett. A 69, 214 (1978).

[12] R. Jullien and P. Pfeuty, Phys. Rev. B 19, 4648 (1979).

[13] I. Peschel and K.D. Schotte, Z. Phys.- Condensed Matter B 54, 305 (1984).

[14] O. Rojas, S. M. de Souza, E. V. Corrêa Silva, and M. T. Thomaz, Phys. Rev. B 72, 172414 (2005).

[15] O. Rojas, S. M. de Souza, E. V. Corrêa Silva, and M. T. Thomaz, Eur. Phys. J. B 47, 165 (2005). 